\title{
CONTRACTING EXTENSIONS AND CONTRACTIBLE GROUPS
}

\author{
BY PETER R. MUELLER-ROEMER ${ }^{1}$
}

Communicated by Kenneth Ross, April 13, 1973

Wiener's classical tauberian theorem has been extended recently to some noncommutative, noncompact groups (see [1], [3], [8] and [10]). Our Theorems 1 and 2 are Wiener type theorems, and interest in them led to the study of contractible groups. It was rather surprising that all contractible Lie-groups are unipotent matrix groups (Theorem 3).

1. Contracting group extensions. A locally compact group $N$ is contractible provided it has enough contractions, i.e., for any compact set $K \subset N$ and any neighborhood $W$ of the identity in $N$, there is a homeomorphic automorphism $h \in$ Aut $N$ such that $h K \subset W$. The ordered pairs $(K, W)$ form a directed set with respect to the relation $\leqq$, defined by $(K, W) \leqq\left(K^{\prime}, W^{\prime}\right)$ if and only if $K \subseteq K^{\prime}$ and $W \supseteq W^{\prime}$. For every $n=(K, W)$ choose a contraction $h_{n}$ with $h_{n} K \subset W$, then $\left\{h_{n}\right\}$ is a net and for any compact set $K \subset N$ we have $\lim _{n} h_{n} K=\{e\}$ (e the neutral element of $N$ ).

A locally compact group $G$ is a contracting extension of its normal subgroup $N$ provided the set of restrictions to $N$ of inner automorphisms of $G$ contains enough contractions of $N$. Thus $N$ must be contractible to admit contracting extensions. For example, if $G \subseteq$ Aut $N$ is a locally compact group and contains enough contractions of $N$, then the semidirect product $G=G$ (s) $N$ is a contracting extension of $N$.

If $G$ is an extension of $N$ and $G=G / N$ is the corresponding factor group we will usually denote their elements respectively by $x, \xi, \dot{x}$, their (left) Haar measures by $d x, d \xi, d \dot{x}$, and their moduli by $\Delta, \delta$ and $\Delta$. We suppose that Weil's formula $d x=d \xi d \dot{x}$ holds.

Let us suppose for a moment that $G$ is separable (i.e. has a countable basis of open sets). Then there exists a measurable cross-section $\sigma$ of $G$ with respect to $N$ (cf. [9]); i.e., there is a measurable function $\sigma: G \cdot \rightarrow G$ with $\sigma(\dot{x}) \in \dot{x}=x N$ and $\sigma(\dot{e})=e$. Suppose further that there is a net $\left\{h_{n}\right\}$ of contractions of $N$ as above, such that $\lim _{n} h_{n}(x)$ exists for locally almost

AMS (MOS) subject classifications (1970). Primary 43A20, 43A65, 22E25, 22E35; Secondary 43A80.

Key words and phrases. Wiener-Tauber theorems.

1 This article summarizes results from the author's dissertation which he prepared under the guidance of Horst Leptin, Bielefeld. The final version with proofs and worked out examples is being prepared for publication in J. Reine Angew. Math. 
all $x$ in $G$ (with respect to $d x$ ). Let $\sigma_{n}(\dot{x})=h_{n}(\sigma(\dot{x})) . \sigma_{n}$ is then a measurable function and $\rho(\dot{x})=\lim _{n} \sigma_{n}(\dot{x})$ exists locally almost everywhere on $G$. Since $G$ is separable it is metrizable, and the net $\left\{h_{n}\right\}$ can be replaced by a sequence. By Egoroff's theorem (cf. [2]) we have the property:

(E) There is a measurable cross-section $\sigma$ of $G$ with respect to $N$ and a measurable function $\rho$ from $G$ into $G$; and for each compact set $K \subset G$. and every $\varepsilon>0$, there is a compact set $K_{1} \subset K$ such that $d \dot{x}\left(K \backslash K_{1}\right)<\varepsilon$ and the restrictions $\sigma_{n} \mid K_{1}$ are continuous and converge uniformly to $\rho$ as functions on $K_{1}$.

From now on we will not use the separability of $G$ but we will suppose that the property $(\mathbf{E})$ holds.

Let $L^{1}(G)$ be the set of all Haar-measurable and absolutely summable complex-valued functions on $G$. With the usual convolution and involution, $L^{1}(G)$ is an involutive Banach algebra, and $L^{\infty}(G)$ is its Banach space dual. $G$ acts weak-*-continuously on $L^{\infty}(G)$ by the usual left and right translations. Subspaces which are closed under these actions are called bi-invariant.

An involutive Banach algebra $B$ is said to have the Wiener property if and only if:

(W) Every proper closed two-sided ideal $I \Delta B$ is contained in the kernel of an irreducible, continuous *-representation of $B$ on some Hilbert space.

$B$ is said to be tauberian if and only if it has the property:

(T) Every proper, closed two-sided ideal $I \Delta B$ is contained in a maximal modular two-sided ideal of $B$.

We will say that a group $G$ is tauberian (or has property $(\mathbf{W})$ ) if $L^{1}(G)$ is tauberian (or satisfies $(\mathbf{W})$ ).

THEOREM 1. Let $G$ be a contracting extension of $N$ satisfying (E). If $G / N$ satisfies $(\mathbf{W})$ or $(\mathbf{T})$, then so does $G$.

The proof of this theorem is based on the following lemma and proposition. Since the canonical projection $p: G \rightarrow G$ is continuous and open and the function $\rho$ is measurable, the composite map $r=\rho \circ p: G \rightarrow G$ is measurable.

LEMMA 1. Let $G$ be a contracting extension of $N$ satisfying $(\mathbf{E})$, and let $M$ be a weak-*-closed, bi-invariant subspace of $L^{\infty}(G)$. If $\phi \in M$ is left uniformly continuous on $G$ then $\phi \circ r \in M$.

Proposition 1. Let $G$ and $M$ be as in Lemma 1, and let $M_{0}$ be the subset of all $\phi \in M$ which are constant on the cosets with respect to $N$. Then $M_{0}$ is a nontrivial, bi-invariant subspace of $M$.

PROPOSITION 1'(DUAL Version). Let $G$ be as above. If $I$ is a proper, 
closed two-sided ideal in $L^{1}(G)$, and if $J$ is the kernel of the morphism $f \rightarrow f \cdot$ of $L^{1}(G)$ onto $L^{1}(G \cdot)$ (where $\left.f \cdot(\dot{x})=\int_{N} f(x \xi) d \xi\right)$, then the closure $\mathrm{cl}(I+J)$ is a proper, closed, two-sided ideal in $L^{1}(G)$; equivalently the closure $\mathrm{cl}(I \cdot)$ of the image of I under the above morphism is a proper closed two-sided ideal in $L^{1}\left(G^{*}\right)$.

2. Some extensions of contractible algebras. Let $A$ be an involutive Banach algebra on which a locally compact group $G$ acts strongly continuously by isometric, involutive, algebra automorphisms $T_{x}$, $x \in G$. The algebra $A$ is $T$-contractible provided that there is a net $\left\{x_{n}\right\}$ in $G$ such that

(i) $\lim _{n}\left(T_{x_{n}} a\right) b$ exists in $A$ for all $a, b \in A$, and

(ii) for some $u \in A$ the net $\left\{T_{x_{n}} u\right\}$ is an approximating unit for $A$.

For example, if $N$ is a contractible group, $A=L^{1}(N)$ and $G=$ Aut $N$ is a locally compact group, then $A$ is $T$-contractible if we define $T$ by $\left(T_{x} f\right)(\xi)=\Delta\left(x^{-1}\right) \cdot f\left(x^{-1}(\xi)\right)$ for $x \in G, f \in A, \xi \in N$. In fact $T_{x_{n}} f$ converges to the scalar $\lambda(f)=\int_{N} f(\xi) d \xi$. Since $A$ contains approximating units, $A$ can be isometrically imbedded in its adjoint algebra $A^{b}$, which is itself an involutive Banach algebra with unit (cf. [7, §3]).

LEMMA 2. Let $A$ be a T-contractible algebra. The equation

$$
R_{a} b=\lim _{n}\left(T_{x_{n}} a\right) b \quad(a, b \in A)
$$

defines an involutive representation $R$ of $A$ into its adjoint algebra $A^{b}$. The kernel $j=\operatorname{ker} R$ of $R$ is $G$-invariant, if the $x_{n}$ belong to the center of $G$.

Let $L=L(G, A ; T)$ be the generalized $L^{1}$-algebra with trivial factor system (cf. $[7, \S 1])$. As a Banach space, $L$ is isomorphic to the projective tensor product $L^{1}(G) \hat{\otimes} A$. The convolution of $f, g \in L$ is defined by the Bochner integral $f * g(x)=\int T_{y} f(x y) \cdot g\left(y^{-1}\right) d y$, and the involution by $f^{*}(x)=\Delta\left(x^{-1}\right) T_{x^{-1}} f\left(x^{-1}\right)^{*}$. L can be viewed as an extension of the algebra $A$ by the group $G$ (cf. [4]).

Suppose $j=\operatorname{ker} R$ is $G$-invariant. Let $A^{\cdot}=A / j$ be the involutive Banach algebra quotient of $A$ by $j$, and define $T \cdot$ on $A$ by $T_{x}(a+j)=$ $\left(T_{x} a\right)+j$. The canonical projection $A \rightarrow A$. induces an isometric isomorphism $L / J \cong L=L\left(G, A ; T \cdot\right.$ ) which we denote (par abuse) by $R_{*}$ (cf. $[7, \S 5])$. The kernel $J$ of $R_{*}$ can be identified with $L^{1}(G) \hat{\otimes} j$.

LEMMA 3. Let $A$ be a T-contractible algebra and assume that $j=\operatorname{ker} R$ is $G$-invariant. Let $J=\operatorname{ker} R_{*}$ be as above.

(i) $\lim _{n}\left(T_{x_{n}} f\right) * g=0$ for all $f \in J$ and $g \in L$, where $\left(T_{x} f\right)(y)=T_{x}(f(y))$.

(ii) Let $p_{i}$ be an approximating unit of $L^{1}(G)$; if $R_{u}=\mathrm{id}_{A}$ for some $u \in A$ and $p_{i, n}=T_{x_{n}}\left(p_{i} \otimes u\right)=p_{i} \otimes T_{x_{n}} u$, then $\left\{p_{i, n}\right\}$ is an approximating unit of $L$, where $(i, n) \geqq\left(i^{\prime}, n^{\prime}\right)$ iff $i \geqq i^{\prime}$ and $n \geqq n^{\prime}$. 
Proposition 2. Let $A$ be a T-contractible algebra and assume that $j=\operatorname{ker} R$ is G-invariant. If $I$ is a proper, closed, two-sided ideal in $L=L(G, A ; T)$ then so is the closure of $I+J$.

By Proposition 2, $L$ will be wienerian (W) or tauberian (T) if $L$ has the respective property.

THEOREM 2. Let $A$ be a $T$-contractible algebra. Let $R$ be as in Lemma 1 , but assume that each $R_{a}$ is a scalar multiple of the identity operator. Assume that $j=\operatorname{ker} R$ is $G$-invariant. If $G$ satisfies $(\mathbf{W})$ or $(\mathbf{T})$ then so does $L=L(G, A ; T)$.

The method of proof in this paragraph is essentially the same as in $[\mathbf{1 0}]$, whereas the method in $\S 1$ is new, and different from the method in [3].

3. Contractible Lie groups and Lie algebras. A few facts about contractible groups in general are collected in

Proposition 3. Let $G$ be a nontrivial contractible group.

(i) $G$ is neither compact nor discrete.

(ii) If $G$ is locally connected, then also globally.

(iii) If $G$ is locally simply connected, then also globally.

(iv) If $G$ has a nontrivial compact subgroup, then it has arbitrarily small ones.

(v) If $G$ has a compact open subset, then $G$ is totally disconnected.

Let $\boldsymbol{K}$ be a nondiscrete, complete field of characteristic 0 , and let $\lambda \rightarrow|\lambda|$ be a norm (= valuation) of $\boldsymbol{K}$. Since $\boldsymbol{K}$ is nondiscrete there are nonzero $\lambda_{n} \in \boldsymbol{K}$ with $\lim _{n}\left|\lambda_{n}\right|=0$. If $M \subset \boldsymbol{K}$ is (norm-) bounded then the diameters of the sets $\lambda_{n} M$ converge to 0 . Multiplication by a scalar $\lambda_{n} \neq 0$, defines an automorphism of $\boldsymbol{K}$ 's additive group. The additive group of $\boldsymbol{K}$ is thus contractible if locally compact.

Let $\mathscr{G}$ be a finite-dimensional Lie algebra over $\boldsymbol{K}$ with Lie product $(x, y) \rightarrow[x, y]$ and norm $x \rightarrow|x|$ for which $|[x, y]| \leqq|x| \cdot|y|$. The norm $|h|$ of a Lie homomorphism $h$ of $\mathscr{G}$ is the norm of $h$ as a linear operator of the normed space $\mathscr{G} ;|h|=\sup \{|h x| ;|x| \leqq 1\}$.

A contraction of the Lie algebra $\mathscr{G}$ is a Lie automorphism $h$ with $|h|<1$. If $\mathscr{G}$ has one contraction $h$, then it has enough contractions and we call $\mathscr{G}$ contractible: the powers $h^{n}$ of $h$ map every bounded set eventually into any 0 -neighborhood of $\mathscr{G}$, because their norms $\left|h^{n}\right|$ converge to 0 .

Proposition 4. Finite dimensional contractible Lie algebras over $\mathbf{K}$ are nilpotent.

Examples. (1) All freely generated, nilpotent Lie algebras are contractible. 
(2) All nilpotent Lie algebras of dimension $\leqq 6$ are contractible, but some are not freely generated. (This last result is based on the classification of these Lie algebras in [11].)

A unipotent matrix over $\boldsymbol{K}$ is an (upper) triangular matrix of finite order with coefficients from $\boldsymbol{K}$ and 1's in the main diagonal. A unipotent group over $\boldsymbol{K}$ is (up to a global isomorphism) a group of unipotent matrices with matrix multiplication as its group operation, which is complete with respect to a norm topology on the respective matrix ring. The topology of a unipotent group does not depend on the choice of norm because $\boldsymbol{K}$ (etc.) is completely metrizable, and Baire's theorem applies.

Proposition 5. If $\mathscr{G}$ is a finite dimensional nilpotent Lie algebra over $\boldsymbol{K}$ (not necessarily contractible) then $\mathscr{G}$ can be imbedded into an associative matrix algebra $A$ over $\boldsymbol{K}$, such that the power series $\exp (x)=\sum_{n \geq 0} x^{n} / n$ !, as evaluated in $A$, reduces to a polynomial for all $x \in \mathscr{G}$, and such that the global image $\exp \mathscr{G}$ of $\mathscr{G}$ under $\exp$ is a unipotent group.

The proof of this proposition depends on the theorems of Ado, Lie and Campbell-Hausdorff (cf. e.g. [5]).

THEOREM 3. If $G$ is a contractible Lie group of finite dimension over the field $\boldsymbol{R}$ of real numbers or the field $\boldsymbol{Q}_{p}$ of $p$-adic numbers, then $G$ is a unipotent group.

In the real case, the proof of Theorem 3 is achieved through Propositions 6 and 7 below, which in turn depend on classical theorems. In the $p$-adic case, however, we rely on results from [6], notably the "inversion of the Campbell-Hausdorff formula" [ibid., IV, 3.2.3].

Proposition 6. The Lie algebra $\mathscr{G}$ of a contractible Lie group $G$ over $\boldsymbol{R}$ is contractible and thus nilpotent.

Proposition 7. If $G$ is a connected and simply connected nilpotent Lie group over $\boldsymbol{R}$ (not necessarily contractible), then $G$ is a unipotent group.

The author would like to thank Horst Leptin for his encouragement and advice.

\section{REFERENCES}

1. W. B. Beiglböck, Some examples of exponential groups with tauberian group algebra, University of Heidelberg, 1972 (preprint).

2. N. Bourbaki, Eléments de mathématique. Fasc. XIII. Livre VI: Intégration. Chap. 4, 2ième éd., Actualités Sci. Indust., no. 1175, Hermann, Paris, 1965, p. 175. MR 36 \#2763.

3. P. Eymard, Moyennes invariantes et représentations unitaires, Lecture Notes in Math., vol. 300, Springer-Verlag, Berlin and New York, 1972, pp. 99-109.

4. J. M. G. Fell, An extension of Mackey's method to Banach*-algebraic bundles, Mem. Amer. Math. Soc. no. 90 (1969). MR 41 \# 4255. 
5. N. Jacobson, Lie algebras, Interscience Tracts in Pure and Appl. Math., no. 10, Interscience, New York, 1962. MR 26 \#1345.

6. M. Lazard, Groups analytiques p-adiques, Inst. Hautes Études Sci. Publ. Math. No. 26 (1965), 389-603. MR 35 \#188.

7. H. Leptin, Verallgemeinerte $L^{1}$-Algebren und projektive Darstellungen lokal kompakter Gruppen. I, II, Invent. Math. 3 (1967), 257-281; ibid. 4 (1967), 68-86. MR 37 \# 5328.

8. - On group algebras of nilpotent Lie groups, Studia Math. 47 (1973).

9. G. W. Mackey, Induced representations of locally compact groups. I, Ann. of Math. (2) 55 (1952), 101-139. MR 13, 434.

10. P. R. Mueller-Roemer, A tauberian group algebra, Proc. Amer. Math. Soc. 37 (1973), $163-166$.

11. M. Vergne, Variété des algèbres de Lie nilpotentes, Thèse, La Faculté des Sciences de l'Université de Paris, June 1966 (preprint).

Department of Mathematics, East Carolina University, Greenville, North CAROLINA 27834 